\title{
Synchronous Power Controller Merits for Dynamic Stability Improvement in Long Line by Renewables
}

\author{
Mostafa Abdollahi, Jose Ignacio Candela, Joan Rocabert, Raul Santiago Munoz Aguilar, and Juan Ramon Hermoso \\ Technical University of Catalonia \\ Barcelona, Spain \\ Mostafa.Bastaki@gmail.com
}

\begin{abstract}
Growing universal demand for greater use of renewable energy resources (RES) installed in dispersed location, has caused to more urgent need for long transmission systems to use these green sources. Small signal stability is one of vital challenges for transmission system operators in presence of big RES. In this paper, a Static Synchronous Generators based in Synchronous Power Controller (SSG-SPC) is presented and its capabilities for dynamic stability enhancement in a long AC transmission system is investigated. Dynamic modelling of a long line and then study in Uncompensated and Compensated cases by using time domain simulation and modal analysis, and then experimental test confirms that SSG-SPC can be effective in active damping of power oscillations and reducing small signal stability challenges.
\end{abstract}

Keywords - Static synchronous generators; Synchronous power controller; Long AC transmission system; Dynamic modelling; Small signal stability

\section{INTRODUCTION}

Renewable Energy Resource (RES) technologies, especially wind power and solar have grown rapidly in the past decade [1]. up to now the main reason for using major part of RES was just following nature and environmental benefits, cost saving and market issues and main advantage of this resources was their local controllability through the use of power electronics devices. But nowadays, some challenges is appeared for power system in presence of high penetration of RES [2]. Impact on small signal stability is one of the main technical challenge. this challenge is more critical especially after growing the rated power of RES and able to have big participation ratio in generation area, as well as working in higher voltage level and connecting directly to the transmission level, because each of them needs to comply with the special grid codes [3].According to variety of primary sources and outspread positions of installed RES in Europe (wind farms in North sea and Mediterranean solar plants in south), as well as their different effect on the power system operation, the super gird idea is one of the best existing solution for reducing this challenge [4]. Moreover, to have better balance between generation and consumption by greater use of RES, having a long transmission line with acceptable stability level is very important to achieve a high performance super gird. When a major part of big traditional synchronous generators (SG) be replaced by new RES technologies which work as non-synchronous generation units, reduction of global rotational inertia and lack of overall damping are the first drawback which will arise in the global power networks and has significant damaging effects on dynamic stability [5]. According to a few studies [5], the best current procedure for this challenge is to mimic the characteristics of a big conventional synchronous generator by RES converters. In this case, such electronic generation units can be called as Static Synchronous Generators (SSG). In SSG concept, some virtual meaning such as Virtual Inertia, Virtual Damping and Virtual Admittance (VA) will be implemented in controller of a voltage source converters (VSC). These concepts are not physical quantity so can be defined and tuned flexible during control process. Synchronous Power Controller (SPC) technique [6] is one of the highly efficient idea among SSG concepts. Synchronizing with the grid without using Phased Locked Loop (PLL) system and at the same time, imposing flexible big virtual damping and virtual inertia in the grid, are the main advantages of this technique which can be very useful for small signal stability enhancement in power system. In this work, after introducing a SSG based in SPC (SSG-SPC), capabilities of this technology for improving dynamic stability in a long $\mathrm{AC}$ transmission system is analyzed. In continue, firstly VA modelling and then grid synchronization by SPC are described. After that, dynamic modelling of a long ac transmission system is expressed in two cases: Uncompensated Line (UCL), and Compensated Line (CL) by SSG-SPC. Then by using time domain simulation in MATLAB/Simulink and modal analysis, and finally by a scaled down laboratory platform, SSG-SPC capabilities for improving small signal stability is investigated.

\section{STATIC SYNCHRONOUS GENERATOR}

\section{A. Virtual Admittance Modelling}

Based on idea suggested in [6], control of a VSC at current mode by using the VA concept can be shown as Fig. 1.

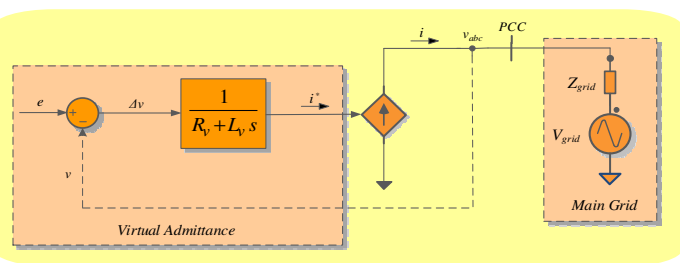

Fig. 1. Virtual admittance idea [6].

The main role of VA is that to provide a current reference for a current controlled VSC. The idea is argued that, first of all the voltage at point of common coupling (PCC) $v$ will be measured. Then, this voltage will compared with an internal electromotive force $e$ as an internal virtual voltage. The error of voltage $\Delta v$ 
will pass through the VA model. Finally current reference $i^{*}$ can be calculated as:

$$
i^{*}(s)=\frac{e(s)-v(s)}{R_{v}+L_{v} s} \cdot \Delta v(s)
$$

\section{B. Grid Synchroniziation by SPC}

Based on SPC idea [6], Electromechanical model (EM) of SG in combination with VA idea, can be used for grid connection of VSC as depicted in Fig. 2. EM part will control active power exchanging between VSC and external grid, and in this way grid phase tracking will be achieved without using PLL. Reactive power control channel will provide reference magnitude $E^{*}$, and finally reference value of internal virtual emf $e^{*}$ will generated for VA model.

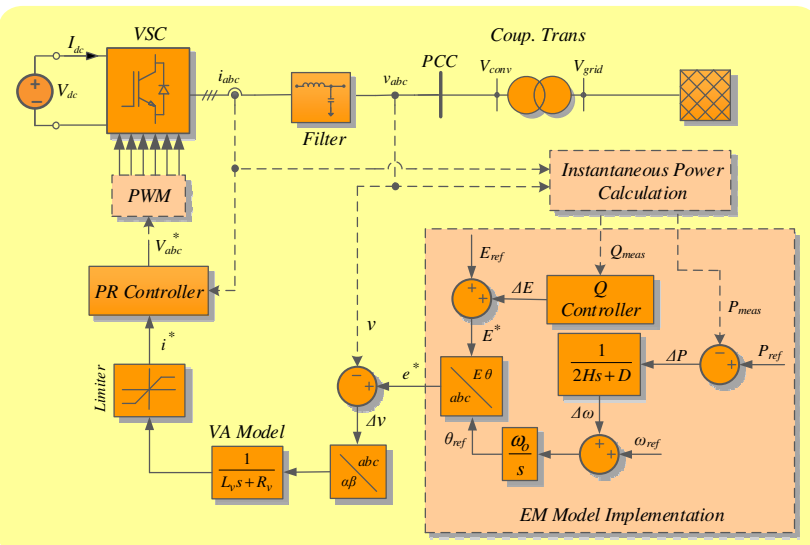

Fig. 2. Grid synchronization of VSC based on SPC idea [6]

If VA model has enough big inductive part and as well as PCC voltage be connected to an external grid by a coupling transformer, overall impedance between VSC and external grid has inductive characteristics $Z \approx X \angle 90^{\circ}$. So, $P$ can be controlled by voltage angle $\delta$, and $Q$ by voltage magnitude, respectively as bellow:

$$
\begin{gathered}
P \approx \frac{E \cdot V}{X} \sin (\delta) \\
Q \approx \frac{E}{X}[E-V \cos (\delta)]
\end{gathered}
$$

\section{LONG LINES DYNAMIC MODELING}

A long AC transmission system for small signal stability analysis is considered as is shown in Fig. 3. In this system, conventional SG as weaker generation area is connected to strong grid through a long line, and local load $P_{L 1}$ is supplied jointly by the line and SG. For more simplicity by using dc power flow, exchanged active power through the line $P_{i j}$ in per unit system, assuming voltages are $1 p u$, can be calculated as below [7]:

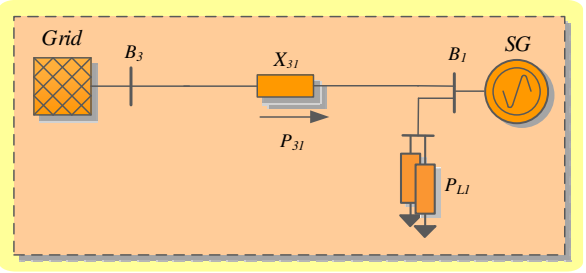

Fig. 3. Long AC transmission system (Uncompensated Line)

$$
P_{i j} \approx \frac{1}{X_{i j}}\left(\theta_{i}-\theta_{j}\right)
$$

Where, $\theta_{i}$ and $\theta_{j}$ are phase angle at Sending-end bus $i$ and Receiving-end bus $j$, respectively. Also, $X_{i j}$ represents reactance of tie line between these buses. Dynamic modelling of this transmission system when is operated as UCL, and also when is working as CL, can be done as follow.

\section{A. Uncompensated Line Modelling}

By considering a second order swing equation (SOSE) to describe dynamics of SG (ignoring PSS effects), dynamic model of UCL, can be depicted as Fig. 4. Where, $P_{31}$ is transferred power from B3 to B1 and can be calculated by (4) when $X_{i j}=$ $X_{31}$ and indicates reactance of all length of line. Also, $H_{1}$ is inertia constant and $D_{1}$ is damping coefficient of SG, respectively. $P_{\text {mech }}$ is mechanical input power of prime mover and $\omega_{o}$ is nominal grid frequency in radians.

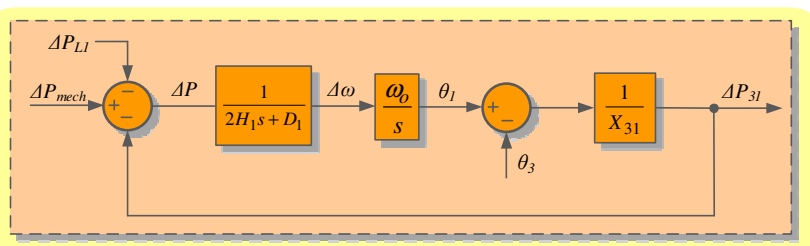

Fig. 4. Dynamic model of uncompensated line connected to SG

Based on this model, transfer function of closed loop system in Laplace domain is:

$$
\frac{\Delta P_{31}}{\Delta P_{\text {mech }}-\Delta P_{L 1}}=\frac{\left(\omega_{o} / X_{31}\right)}{2 H_{1} s^{2}+D_{1} s+\left(\omega_{o} / X_{31}\right)}
$$

Comparing characteristic equation of system (5), with standard form of second-order system in normal operating point with damping ratio $\xi$ less than one, gives the roots as bellow:

$$
s_{1,2}=-\frac{D_{1}}{4 H_{1}} \pm J \frac{\omega_{o}}{2 H_{1}} \sqrt{\frac{2 H_{1}}{\omega_{o} X_{31}}-\frac{D_{1}^{2}}{4}}
$$

\section{B. Compensated Line Modelling}

Based on several studies [8], middle point installation is the best option for optimal compensation of a long line. So, here as base case, CL is considered as is shown in Fig. 5 where SSG$\mathrm{SPC}$ is connected to B2. 


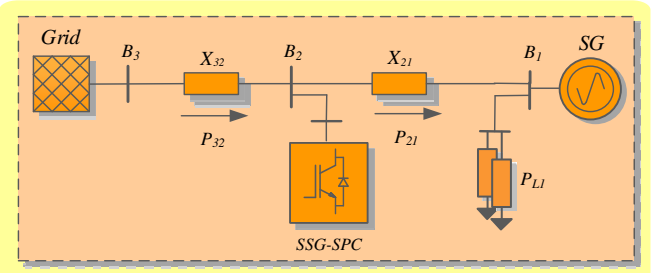

Fig. 5. Long transmission line compensated by SSG-SPC.

In this case, $P_{21}$ and $P_{32}$ indicate active power in Receivingend and Sending-end buses, respectively. As well as, $X_{32}=$ $X_{21}=\left(X_{31} / 2\right)$. Considering SOSE for both SG and SSG-SPC, dynamic modelling of CL can be displayed by Fig. 6. Where, $\mathrm{H}_{2}$ and $D_{2}$ are the virtual inertia constant and virtual damping coefficient of SSG-SPC, respectively. As well as, $\theta_{1}$ to $\theta_{3}$ are phase angle in B1 to B3. After doing some mathematical operation, characteristic equation of closed loop system from $\Delta P_{L 1}$ to $\Delta P_{31}$ can be write as:

$$
a_{4} s^{4}+a_{3} s^{3}+\left(a_{21}+a_{22}\right) s^{2}+a_{1} s^{1}+a_{0}=0
$$

Where,

$$
\begin{gathered}
a_{4}=2 H_{1} * 2 H_{2} * X_{21} X_{32} \\
a_{3}=\left(2 H_{1} D_{2}+2 H_{2} D_{1}\right) * X_{21} X_{32} \\
a_{21}=\left(D_{1} D_{2} X_{21} X_{32}\right) \\
a_{22}=\left(2 H_{2} X_{32}+2 H_{1} X_{21}+2 H_{1} X_{32}\right) * \omega_{o} \\
a_{1}=\left(D_{2} X_{32}+D_{1} X_{21}+D_{1} X_{32}\right) * \omega_{o} \\
a_{0}=\omega_{o}^{2}
\end{gathered}
$$

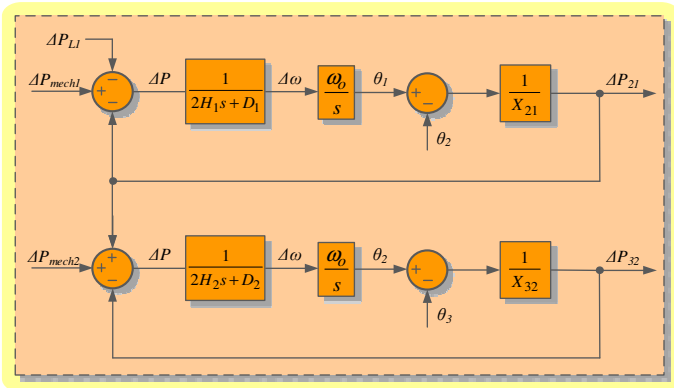

Fig. 6. Dynamic model of compensated line with SSG

For more simplicity and as well as having better analysis about the effect of SSG-SPC on overall dynamic behavior of CL, all Power System Stabilizers (PSS) is considered to be deactivated. So, since the internal damping of SG are very small [7], $D_{1}$ can be considered equal to zero. By this assumption and also convert (7) to standard representation format of a 4th order equation we have new coefficient as bellow:

$$
\begin{gathered}
a_{4}^{\prime}=1 \quad a_{3}^{\prime}=\frac{D_{2}}{2 H_{2}} \quad a_{21}^{\prime}=0 \\
a_{22}^{\prime}=\left(\frac{H_{2} X_{32}+H_{1}\left(X_{21}+X_{32}\right)}{2 H_{1} H_{2} X_{21} X_{32}}\right) * \omega_{o} \\
a_{1}^{\prime}=\frac{D_{2} \omega_{o}}{2 H_{1} * 2 H_{2} * X_{21}} \quad a_{0}^{\prime}=\frac{\omega_{o}^{2}}{2 H_{1} * 2 H_{2} * X_{21} X_{32}}
\end{gathered}
$$

According to the $a_{4}^{\prime}$ to $a_{0}^{\prime}$ some preliminary forecast about SSG-SPC effect on dynamic model of CL can be done: i) Overall damping of the system is caused only by $D_{2}\left(a_{3}^{\prime}, a_{1}^{\prime}\right)$, ii)
Global constant inertia is combination of SG and SSG-SPC effects and also will be affect by location of SSG-SPC $\left(a_{22}^{\prime}\right)$, iii) Frequency of oscillation will change by variation in $D_{2}$ and also location of SSG-SPC $\left(a_{22}^{\prime}, a_{1}^{\prime}, a_{0}^{\prime}\right)$ and iv) Location of SSG-SPC will affect the overall damping of system $\left(a_{1}^{\prime}\right)$.

\section{SSG-SPC CAPABILTIES FOR SMALl SigNAL STABILITY}

Both UCL and CL models are simulated by MATLB/ Simulink for evaluation SSG-SPC capability in dynamic stability improvement. A step change in local load $\Delta P_{L 1}=$ $0.5 p u$ is applied then Sending-end and Receiving-end power are analyzed. As well as, eigenvalues are extracted in each study by using (7) to confirm simulation results.

\section{A. Variable Damping in SSG-SPC}

Flexibility in $D_{2}$ is one of the main SSG-SPC capability for control global damping of system. This selection can be done purposefully, meaning that firstly it can be selected to have a proper internal damping ratio in SSG (for example to have $\left.\xi_{S S G}=0.7\right)$ and then it can be modified to have better global damping on system. To clear this feature, simulation results of $\mathrm{UCL}(\mathrm{SG})$ and CL (SG+SSG) for $H_{1}=3.7 \mathrm{~s}, D_{1}=0, H_{2}=5 \mathrm{~s}$, $D_{2}=D, X_{31}=0.75 p u$, and $X_{32}=X_{21}=X_{31} / 2$, are shown in Fig. 7 and eigenvalues for selected magnitude of $D$ are reported in Table I. As can be seen, in UCL due to deactivation of PSS and also $D_{1}=0$, response is undamped oscillatory. As well as from (6), UCL model has two pure imaginary eigenvalues which are located on the $j \omega$ axes and frequency of oscillation is 1.197 $\mathrm{Hz}$. But on the contrary, in CL even when the SSG parameters are designed independently to have low internal damping $\left(D_{2}=\right.$ $\left.78.47, \xi_{S S G}=0.44\right)$, modes which are strongly linked to SG and UCL model, have considerable damping. Power oscillations will damped more by proper increasing in $D$ and in worst case during $2.7 \mathrm{sec}$ system will arrive to new steady state operating point. Deeper investigation of $P_{21}$ and $P_{32}$ shows that, although by increasing $D$, oscillation between $\mathrm{B} 2$ and $\mathrm{B} 3$ will be reduced faster and when $D=177$ we have not big overshoot, but select very big $D$ can lead to new inter area oscillation between B2 and B1. So, it will be very useful to investigate CL modes when $D$ is variable. Poles positions of CL for $D=K_{d}$ and $1 \leq K_{d} \leq 200$ with $\Delta K_{d}=5$, is shown in Fig. 8 . As can be seen, in beginning SG modes will take more damping along the improving damping in SSG modes. Although, the oscillation frequency of SSG modes also will be affected but finally overall damping will be enhanced properly. Internal investigation of SSG shows that $\xi_{S S G}=1$ will occur when $K_{d}=177.25$, which means in this value SSG has an overdamped dynamics. It seems after this critical damping value, SSG cannot have significant interaction with other part of system in CL to be able participate properly in damping of oscillation. Moreover, when SSG is connected to network and provides initially damping for CL, this critical point will occur sooner $\left(K_{d}=155\right)$. So after this internal breaking point, SSG as rigid system has critical poles from system point of view and by increasing more $K_{d}$ firstly oscillations with new frequency will be made and then overall damping of system will be reduced. Beginning of this new oscillation can be seen clearly in $P_{21}$ when $D=177$ (Fig. 7). 


\section{B. Several Location of SSG-SPC}

In this section, effect of SSG-SPC location on small signal stability of CL is investigated. Internal parameters of SSG-SPC is considered to be fixed $\left(D_{2}=124.07, H_{2}=5 s\right)$. By assuming $X_{21}=K X_{31}$ and $X_{32}=(1-K) X_{31}$ when $0 \leq K \leq 1$, SSG located at several positon along line can be modeled. So, smaller $K$ means locations closer to SG. Dynamics models responses and eigenvalues for $K=0.25, K=0.5$ and $K=0.75$ are presented in Fig. 9 and Table II. As can be seen, smaller $K$ leads to response with very lower overshoot, no considerable swing and very fast $(1.75 \mathrm{~s})$ damped at $P_{32}$. Reason is that, when location is close to disturbance, according to fast reaction capability of SSG and smaller electrical distance, SSG is able to participate even in very small swings and in this way swing outspread will be prevented over big part of line. Early small swing with higher frequency in $P_{21}$ for when $K=0.25$, and very soft transient in $P_{32}$ at the same time confirms this reason.
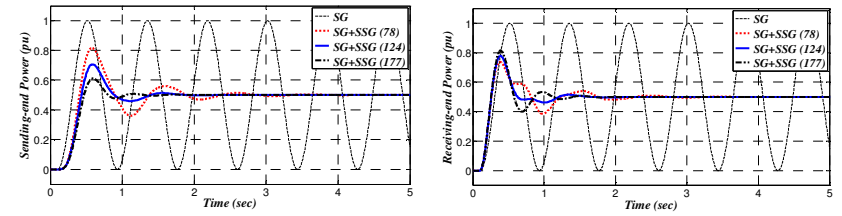

Fig. 7. Sending and receiving active power for several damping

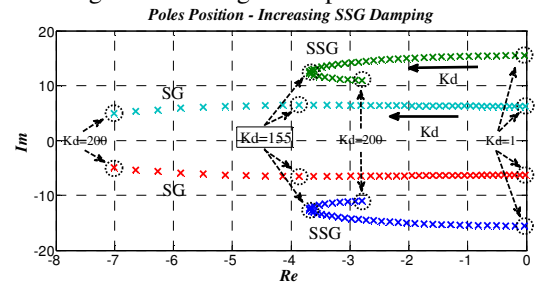

Fig. 8. Impact of SSG-SPC damping variation on system eigenvalues

Table I. Eigenvalues of UCL and CL - Several damping

\begin{tabular}{|c|c|c|}
\hline \multicolumn{3}{|c|}{ Eigenvalues } \\
\hline U & \multicolumn{2}{|c|}{$0 \pm 7.524 i$} \\
\hline \multirow{3}{*}{ 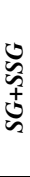 } & $\begin{array}{c}D=78.47 \\
\xi_{S S G}=0.44\end{array}$ & $\begin{array}{l}-2.391 \pm 14.765 i \\
-1.532 \pm 6.328 i\end{array}$ \\
\hline & $\begin{array}{c}D=124.07 \\
\xi_{S S G}=0.70\end{array}$ & $\begin{array}{l}-3.452 \pm 13.488 i \\
-2.752 \pm 6.431 i\end{array}$ \\
\hline & $\begin{array}{l}D=177.25 \\
\xi_{S S G}=1.00\end{array}$ & $\begin{array}{l}-3.271 \pm 11.359 i \\
-5.591 \pm 6.051 i\end{array}$ \\
\hline
\end{tabular}

By increasing $K$, two event will occur. Firstly, changing in phase angle will be sensed by SSG with more delay as result of bigger electrical distance from disturbance place; secondly SSG effectiveness on disturbance will be reduced due to bigger reactance of tie line. That is why, for $K=0.75$ after first big peak oscillation starts to damped and moreover damping duration is longer (7s). In addition, according to lower effect of SSG, frequency of oscillation is imposed by SG modes, as well as disturbances are spread in total length of line and thus we have similar oscillations at receiving and sending end buses. Variation in eigenvalues for $0.1 \leq K \leq 0.9$ when $\Delta K=0.05$ is plotted in Fig. 10. As would be expected, SG modes moves closer to $j \omega$ axes by increasing $K$ which means having lower damping and converting to dominant poles for overall system. But on the other side, SSG modes will get more damping by increasing $K$ due to the location closer to infinite bus and has smaller interaction with oscillation of weaker area. In this plot it can be seen that, overall damping will be close to zero for big $K$. Although, damping ratio of $\mathrm{SG}$ modes $\xi_{S G}$ and thus overall damping will be improved by decreasing $K$ but enhancement in damping ratio $\left(\Delta \xi_{S G}\right)$ is not linear. In areas close to B3 $(0.8 \leq$ $K \leq 0.9), \Delta \xi_{S G}$ is not very big, but in central areas $(0.5 \leq K \leq$ $0.75)$ this variation is significant. Finally, after crossing from middle of line $(K=0.5), \Delta \xi_{S G}$ will be small again. The reason can be expressed by attention to the variation in SSG modes. In $(0.8 \leq K \leq 0.9)$, variation in $\mathrm{SSG}$ damping ratio $\left(\Delta \xi_{S S G}\right)$ is small due to being too close to external strong grid. After that, $\Delta \xi_{S S G}$ is better by moving away from B3 and will affect $\xi_{S G}$ significantly up to $K=0.45$. At this critical point the dominant poles of system will changes from SG modes to SSG modes. So, from this point onwards overall behavior of system is imposed by SSG.

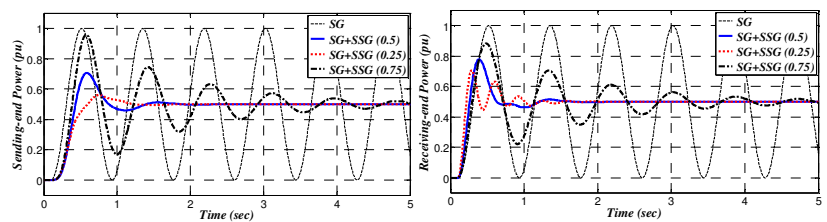

Fig. 9. Impact of SSG-SPC location on power oscillation

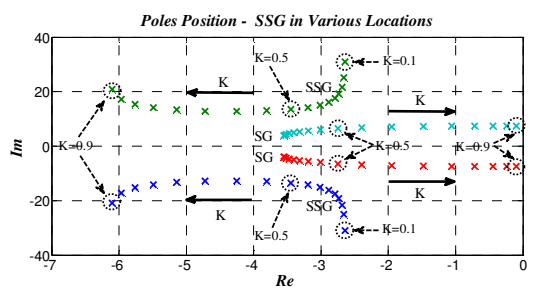

Fig. 10. System modes affected by installation location of SSG-SPC

Table II. Eigenvalues of UCL and CL for different location

\begin{tabular}{|c|c|c|}
\hline & \multicolumn{2}{|c|}{ Eigenvalues } \\
\hline ڤ & \multicolumn{2}{|c|}{$0 \pm 7.524 i$} \\
\hline \multirow{3}{*}{ 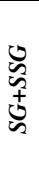 } & $K=0.25$ & $\begin{array}{c}-2.734 \pm 19.261 i \\
-3.470 \pm 4.623 i\end{array}$ \\
\hline & $K=0.5$ & $\begin{array}{c}-3.452 \pm 13.488 i \\
-2.752 \pm 6.431 i\end{array}$ \\
\hline & $K=0.75$ & $\begin{array}{c}-5.486 \pm 14.052 i \\
-0.718 \pm 7.420 i\end{array}$ \\
\hline
\end{tabular}

After this critical point, $\Delta \xi_{S S G}$ is not as big as before and in continue very big changes will happen in frequency of SSG modes. As results, SG modes will also affected by this frequency variation and finally new oscillation with bigger frequency will appear in system (such as oscillation for $K=0.25$ in Fig. 9). It can be concluded that, for this specified SSG, central areas of long line $(0.5 \leq K \leq 0.7)$ are best places to install which will lead to have sufficient effect on dynamic stability without causing any new inter-area oscillation.

\section{SSG-SPC Participation Level}

Effect of SSG-SPC power ratio in providing active power, when SSG-SPC installed at fixed location, will be analyzed in this section. According to dynamic modelling and assuming $\left(2 \mathrm{H}_{2} \mathrm{~S}+\mathrm{D}_{2}\right) * K_{p}$, SSG-SPC contribution can be controlled by $K_{p}$. Bigger $K_{p}$ means SSG-SPC is emulating behavior of a bigger $\mathrm{SG}$ and vice versa. In this study, $D_{2}=124.07$ and $H_{2}=$ $5 s$, are selected as base value and SSG-SPC is installed at middle of line $\left(X_{32}=X_{21}=X_{31} / 2\right)$. If $P_{S S G}^{\text {base }}$ represents base 
value of power ratio, then new power ratio can be expressed as $P_{S S G}^{n e w}=K_{p} * P_{S S G}^{\text {base }}$. The $\mathrm{CL}$ and UCL responses to $\Delta P_{L 1}$ are shown in Fig. 11, when $\Delta K_{p}= \pm 0.5$. As can be seen, by reducing 50\% of $P_{S S G}$ and thus lower effect of SSG-SPC on total active power, in fact $S G$ with very small damping will get major contribution, so finally overall damping will be weaker. As reported in Table III, for $K_{p}=1$, SSG modes with $\xi_{S S G}=0.248$ and SG modes with $\xi_{S G}=0.393$ are very close together, so in this case all of them are dominant poles for CL and will provide large enough damping jointly. When $K_{p}=0.5$, while SSG modes has a bit larger damping ratio $\left(\xi_{S S G}=0.259\right)$, but now SG modes with $\xi_{S G}=0.168$ are new dominant poles and new $\xi_{S G}$ is 2.5 times smaller than previous. So, having smaller overall damping in CL for $K_{p}=0.5$ is reasonable compared to when $K_{p}=1$.
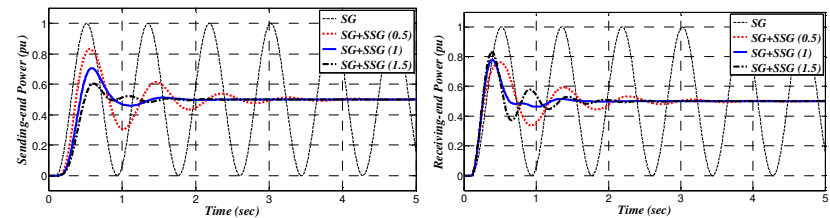

Fig. 11. Impact of SSG-SPC power ratio on power oscillation

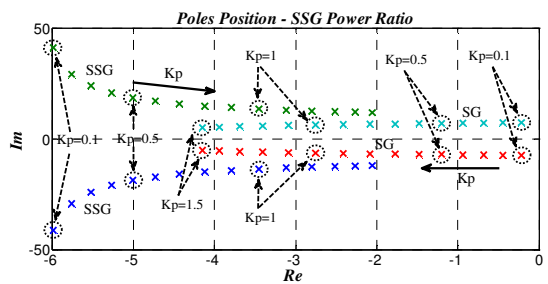

Fig. 12. System modes affected by SSG-SPC power ratio

Table III. Eigenvalues - Several power ratio

\begin{tabular}{|c|c|c|c|c|}
\hline & \multicolumn{2}{|c|}{ Eigenvalues } & $\xi_{S G}$ & $\xi_{S S G}$ \\
\hline U & \multicolumn{2}{|r|}{$0 \pm 7.524 i$} & 0 & - \\
\hline \multirow{3}{*}{ 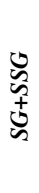 } & $K p=0.5$ & $\begin{array}{c}-5.004 \pm 18.668 i \\
-1.200 \pm 7.025 i\end{array}$ & $\overline{0 .}$ & $\begin{array}{c}0.259 \\
-\end{array}$ \\
\hline & $K p=1$ & $\begin{array}{l}-3.452 \pm 13.488 i \\
-2.752 \pm 6.431 i\end{array}$ & $\begin{array}{c}- \\
0.393\end{array}$ & $\begin{array}{c}0.248 \\
-\end{array}$ \\
\hline & $K p=1.5$ & $\begin{array}{c}-2.054 \pm 11.996 i \\
-4.149 \pm 5.047 i\end{array}$ & $\overline{-}-\overline{635}$ & $\begin{array}{c}0.169 \\
-\end{array}$ \\
\hline
\end{tabular}

However, comparing results of CL for $K_{p}=0.5$ with UCL, shows that even by small participation of SSG-SPC, overall damping of system can be improved dramatically. For CL with $K_{p}=1.5$, although SSG modes are more prevail, but SG modes has also major effect on system response (due to smaller difference between real parts of this two group modes compare to when $\left.K_{p}=0.5\right)$. So compare to base case $\left(K_{p}=1\right)$, although a small reduction in $\xi_{S S G}\left(\Delta \xi_{S S G}=-0.079\right)$ has afflicted the oscillation, but $\Delta P_{S S G}=+50 \%$ gives a considerable damping ratio to $S G$ modes $\left(\xi_{S G}=0.635\right)$. Finally this modifications in system modes will results in oscillation with smaller first overshoot in sending-end bus and as well as active power oscillation with slightly smaller damping in receiving-end bus. Variation in system modes positions for $0.1 \leq K_{p} \leq 1.5$ and $\Delta K_{p}=0.1$ is plotted in Fig. 12, and confirms this analysis.

\section{EXPERIMENTAL TEST}

To experimental validation of theoretical section, a case study of transmission system with details reported in Table IV is implemented in laboratory platform as is shown in Fig. 15. Long line system is modeled in Real-Time Digital Simulator environment (OPAL-RT5600), and three phase voltage of B2 scaled down and then used for reference of PUISSANCE PCU 21KVA amplifier. So this amplifier emulate characteristics of B2 in long line model. A $5 \mathrm{kVA}, 400 \mathrm{~V}, 10 \mathrm{kHz}$ converter which is supplied by a $32 \mathrm{~kW}$ REGATRON DC power supply is connected to amplifier as a SSG-SPC. SPC based controller which is implemented in dSPACE 1103, will control the converter. By measuring three phase exchanged current between amplifier and real converter and then transfer it to inside of OPAL-RT model by proper gain, the behavior of a real SSGSPC will be modelled in middle bus of mentioned long line system. As sample, CL with several damping values $\left(D_{2}=5\right.$ and $\left.D_{2}=124\right)$ are tested by this platform and experimental results are shown in Fig. 13 and Fig. 14.

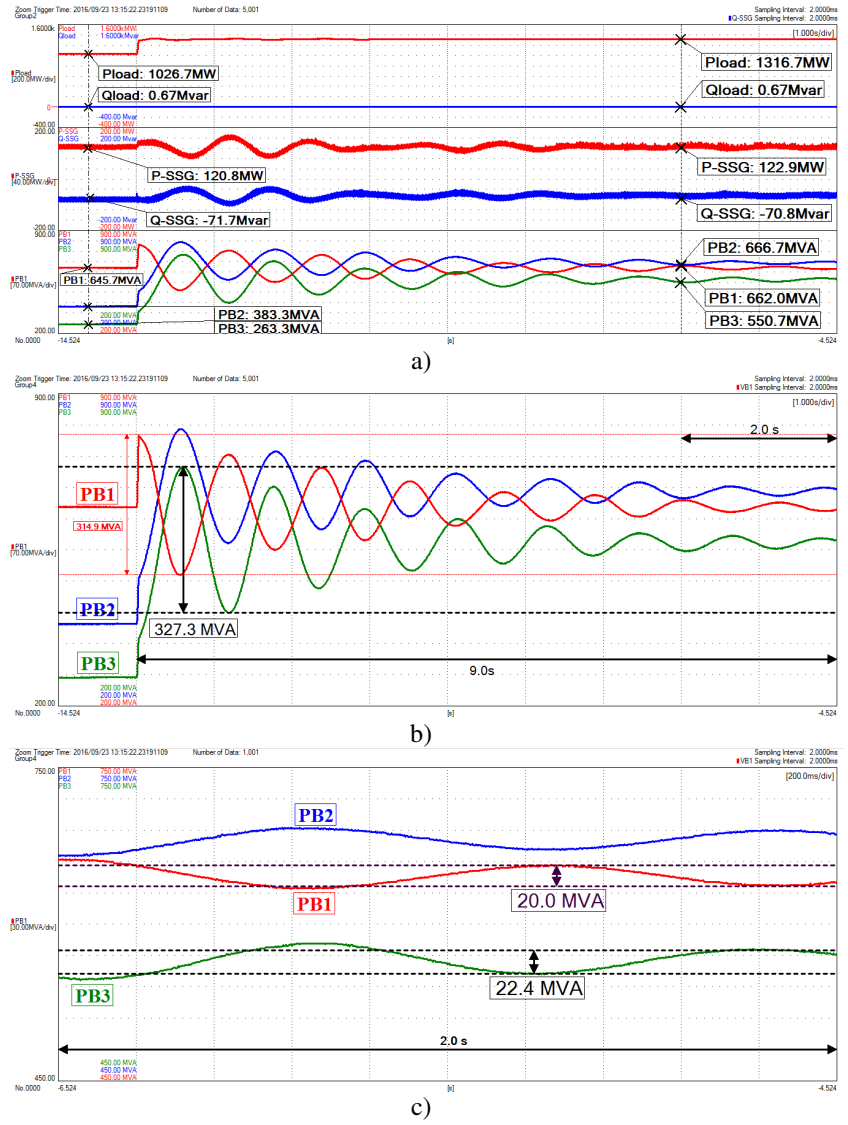

Fig. 13. Response of transmission system for $D_{2}=5$, a) load, SSG-SPC and buses powers, b) oscillation in active power of buses, c) boundary of power oscillation in last 2 seconds.

A base active load $P_{L 1}=1026 M W$ and similar SSG-SPC participation level $P_{S S G}=121 \mathrm{MW}$ is considered at primary steady state condition for both cases. As well as, $P B_{3}=$ $264 M W$ and $P B_{1}=645 M W$ are fixed, which means SSG-SPC has $18.7 \%$ participation ratio in generation compare to $\mathrm{SG}$ generation level. For investigate transmission system response a 
step change in active load $\Delta P_{L 1}=290 M W$ is applied for both case. As can be seen in Fig. 13, for $D_{2}=5$ as small damping effect of SSG-SPC, oscillation in all powers remains for long time (more than $9 s$ ) as well as will affect $P_{S S G}$. Oscillation of sending-end $\left(P B_{3}\right)$ and receiving-end $\left(P B_{1}\right)$ power in last two seconds are 22.4MW and $20 M W$, respectively. But in $D_{2}=$ 124 as is shown in Fig. 14, transferring of oscillatory modes from one area to another is prevented significantly by having more damping in SSG-SPC. Where, amplitude of oscillation in $P B_{3}$ is reduced remarkable from first cycle $\left(\triangle P B_{3}^{D=5}=\right.$ 327.3MW and $\triangle P B_{3}^{D=124}=224.2 M W$ ), while magnitude of oscillation in $P B_{1}$ is fixed on same cycle $\left(\triangle P B_{1}^{D=5}=\right.$ $\left.\Delta P B_{1}^{D=124}=314.9 M W\right)$. Moreover, all oscillation in this case are damped 1.50 times faster than previous case $\left(\triangle P B_{1}^{D=5}=\right.$ $20 M W$ at $\Delta t=8.28 s$ and $\Delta P B_{3}^{D=5}=22.4 M W$ at $\Delta t=8.22 s$ comparing with $\Delta P B_{1}^{D=124}=20 M W$ at $\Delta t=5.65 \mathrm{~s}$ and $\Delta P B_{3}^{D=124}=22.4 M W$ at $\Delta t=5.10 \mathrm{~s}$, where $\Delta t$ shows time interval after $\Delta P_{L 1}$ connection).

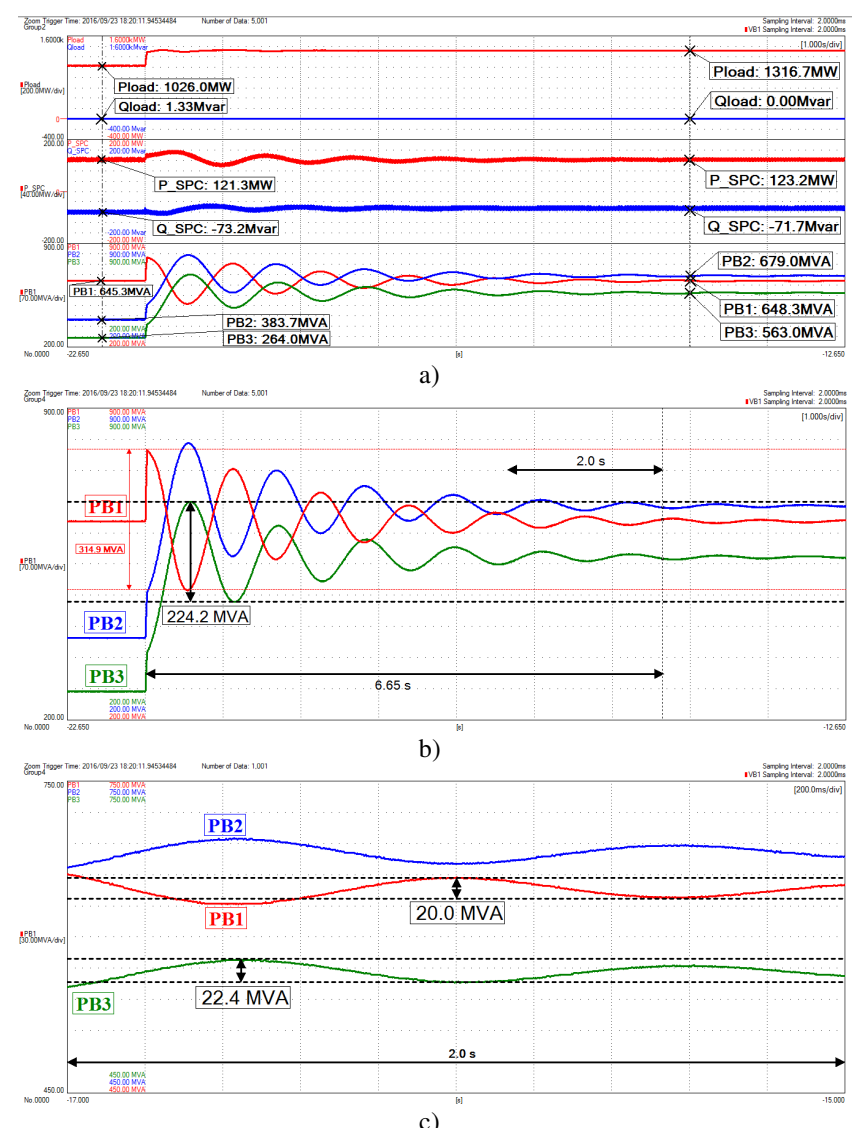

Fig. 14. Response of transmission system for $D_{2}=124$, a) load, SSG-SPC and buses powers, $b$ ) oscillation in active power of buses, c) boundary of power oscillation in mentioned 2 seconds interval on part (b).

\section{CONCLUSION}

In this work, aims to dynamic stability enhancement, capabilities of a Static Synchronous Generator equipped with Synchronous Power Controller (SSG-SPC) is investigated as a highly efficient technology for integration renewables into long
AC transmission system. Dynamic modelling of compensated line shows that, a) possibility of having big and variable virtual damping, as well as b) flexibility in power participation rates, are two main freedom degrees at SSG-SPC which by affecting dominant poles will cause to adding significant overall damping and control of inter-area oscillation. Moreover, optimal placement studies approves that by choosing an appropriate mounting location for SSG-SPC (as a bus with stiff dynamics), will result to prevent spreading of disturbances to other areas. Finally it can be concluded that SSG-SPC could be a useful practical technology for dynamic stability enhancement only by relying on its controller capabilities.

\section{ACKNOWLEDGMENT}

This work was supported by the Spanish Ministry of Science under Project ENE2013-48428-C02-2-R.

Table IV. Case Study Data

\begin{tabular}{|c|c|c|}
\hline \multicolumn{3}{|c|}{ Long Line AC Transmission System } \\
\hline Grid & \multicolumn{2}{|c|}{$5000 \mathrm{MVA}, 500 \mathrm{kV}, 50 \mathrm{~Hz}$} \\
\hline \multirow{2}{*}{ Line } & $r=0.01755 \Omega / \mathrm{km}$ & $l=0.8737 \mathrm{mH} / \mathrm{km}$ \\
\cline { 2 - 3 } & $c=13.33 \mathrm{nF} / \mathrm{km}$ & lentgh $=700 \mathrm{~km}$ \\
\hline \multirow{3}{*}{$S \boldsymbol{G}$} & $1000 \mathrm{MVA}, 13.8 \mathrm{KV}, 50 \mathrm{~Hz}, Q_{\max }=250 \mathrm{Mvar}$ \\
\cline { 2 - 3 } & \multicolumn{2}{|c|}{$X_{d}=1.035 \mathrm{pu}, X_{q}=0.474 \mathrm{pu}, H=3.7 \mathrm{~s}$} \\
\cline { 2 - 3 } & \multicolumn{2}{|c|}{ Trans: $X_{T r}=0.12 \mathrm{pu}, R_{T r}=0.002 \mathrm{pu}$} \\
\hline \multirow{2}{*}{ SSG- } & \multicolumn{2}{|c|}{$5 \mathrm{kVA}, V_{a c}=400 \mathrm{~V}, V_{d c}=600 \mathrm{~V}, 10 \mathrm{kHz}$} \\
\cline { 2 - 3 } SPC & $H_{2}=5 \mathrm{~s}, D_{2}=124, R_{v}=0.1 \mathrm{pu}, X_{v}=0.3 \mathrm{pu}$ \\
\hline
\end{tabular}

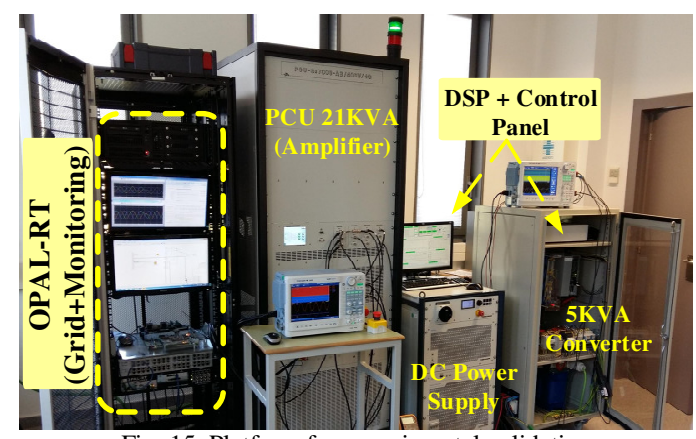

Fig. 15. Platform for experimental validation

\section{REFERENCES}

[1] The European offshore wind industry - key trends and statics 1st half 2015, the European Wind Energy Association (EWEA), July 2015.

[2] J. Quintero, V. Vittal, G. T. Heydt and H. Zhang, "The Impact of Increased Penetration of Converter Control-Based Generators on Power System Modes of Oscillation," in IEEE Transactions on Power Systems, vol. 29, no. 5, pp. 2248-2256, Sept. 2014.

[3] IEEE Standard for Interconnecting Distributed Resources With Electric Power Systems, IEEE Std. 1547-2003, 2003.

[4] J. Blau, "Europe plans a north sea grid," IEEE Spectrum, vol. 47, no. 3, pp. 12-13, 2010.

[5] H. Bevrani, T.Ise, Y.Miura, "Virtual synchronous generators: A survey and new perspectives", Int. J. Electr. Power Energy Syst, Vol. 54, pp. 244254,2014

[6] P. Rodriguez, I. Candela and A. Luna, "Control of PV generation systems using the synchronous power controller," 2013 IEEE Energy Conversion Congress and Exposition, Denver, CO, pp. 993-998, 2013.

[7] P. Kundur, 'Power System Stability and Control', N.Y: M-Hil, 1994.

[8] T. Moger, T. Dhadbanjan, "A novel index for identification of weak nodes for reactive compensation to improve voltage stability", IET Generation, Transmission \& Distribution, vol. 9, no. 14, pp. 1826-1834, 2015. 